\title{
The pathogenesis of experimental periventricular cerebral necrosis and its possible relation to the periventricular leucomalacia of birth trauma
}

\author{
ARTUR ABRAMOWICZ \\ From the Neurology Service, Veterans Administration Hospital, \\ Boston, Massachusetts, U.S.A.
}

Necrotic cerebral softenings located in the periventricular white matter occur sometimes in the brains of patients with occlusive cerebral vascular disease (Greenfield, 1958; Zülch, 1961).

Lesions of the same type are found, however, more frequently in the brains of infants subjected to anoxia or other damaging factors acting before, during, or shortly after birth, and in those of older children or adults with a history of cerebral palsy (Norman, 1949; Benda, 1952; Norman, 1958). Perinatal anoxia is generally thought to represent the main type of 'birth injury' related to the subsequent development of the syndrome of cerebral palsy (double athetosis, spastic diplegia, mental deficiency, seizure states).

In the cases reported, periventricular necroses sometimes co-exist with other cerebral lesions; status marmoratus of the basal ganglia and of the cerebral cortex, granular atrophy, and other cortical scleroses (Courville, 1953; Norman and Urich, 1959). The necrotic lesions of the white matter might range in their appearance from early coagulation necrosis (Banker and Larroche, 1962) to cystic degeneration of the white matter (Benda, 1952). Norman (1958), among other investigators, considered the periventricular softenings the most characteristic central lesions of birth trauma. Banker and Larroche (1962) have found them in $19 \%$ of all the infants who died under 1 month of age.

The aetiopathogenesis of such periventricular encephalomalacia is, up to the present date, poorly understood. Suggested causes, which have included prenatal infection ('congenital encephalitis' of Virchow), venous infarction, ischaemia, have been discussed by several authors (Virchow, 1867;

${ }^{1}$ This work was carried out during the tenure of special traineeship BT-518 from the National Institute of Health, Bethesda, Maryland, held during the academic year 1960-61 at Joseph P. Kennedy Jr. Memorial Hospital and Tufts University School of Medicine, Boston, Massachusetts.
Schwartz, 1961; Norman and Urich, 1959; Banker and Larroche, 1962) but an experimental reproduction of typical periventricular necrosis has not been reported in the literature. Induced anoxia in dogs and other animals resulted sometimes in lesions of the white matter. These, however, differ in many details from the periventricular malacia seen in most human disease states found in conjunction with atherosclerosis and birth injury.

This study is a part of a more extensive experimental investigation of haemodynamic factors participating in the pathogenesis of cerebral border zone lesions in foetal and adult brains undertaken in 1961. In the course of these experiments the brains of several of the animals (cats) presented a selective involvement of the periventricular white matter by multiple and well-defined patchy infarcts. These observations are reported here because of the close resemblance of experimentally produced lesions to the softenings described in human neuropathological material. The haemodynamic factor was clearly implicated in these pathological changes since the lesions were produced by means of arterial occlusion or stenosis with resulting curtailment of the cerebral blood flow.

In the following discussion an attempt at a pathogenetic interpretation is made; it is based upon the available experimental anatomical and physiological data.

Because of its cerebral vascular structure, which closely resembles that seen in the human foetus in the last months of intra-uterine life (Klosovskij, 1951 and 1956), the cat was used as a laboratory animal in this series of experiments.

\section{METHODS}

The experiments were performed with aseptic precautions on healthy anaesthetized mature cats. Nembutal sodium anaesthesia, $25 \mathrm{mg}$. per $\mathrm{kg}$. of body weight in the fasting state, was routinely administered by a single intra- 
peritoneal injection. Those animals which developed post-operative complications of any type were discarded from the subsequent study.

The animal's trachea was intubated with a stiff piece of polyethylene tubing. The basilar artery was exposed through a neck incision followed by trephination of the base of the skull between the tympanic bullae. The dura mater was incised and the basilar artery obliterated by means of a fine silk ligature. Although recovery from this operation was usually complete over a period of one to two days, the animal was kept under observation for at least five additional days.

With the animal fully recovered, the neck wound was re-opened and both common carotid arteries were carefully separated from the surrounding tissues and the accompanying nerve. A 4-0 silk ligature was placed on each of the arteries and tied down so as to constrict or totally obliterate the lumen to a degree varying from one animal to another. The wound was then sutured with the usual technique.

Post-operatively the animals were followed with frequent neurological examinations for periods varying from one week to six months.

Following the individually allowed period of observation, the cat was sacrificed by an overdose of Nembutal. The brain and the ligated segments of the carotid arteries were immediately removed and fixed in $10 \%$ formalin for two or more weeks.

The whole brain was embedded in celloidin or in paraffin and cut in serial gapless sections $20 \mu$ thick. Approximately every twenty-fifth section was stained for myelin sheaths by Loyez' or Weigert's method and every next adjacent section was stained for nerve cells by the Nissl or Bielschowsky-Plien techniques. Other neighbouring sections were electively stained with haematoxylin and eosin, Wiegert's elastic tissue stain, P.T.A.H., and P.A.S.

The ligated segments of the common carotid arteries were also embedded and cut in serial transverse sections and stained with haematoxylin and eosin.

The material was studied with dissecting and compound microscopes. The histopathology of the lesions was described and the patterns of their topographic distribution were reconstructed on template diagrams. The lumina of the ligated carotid arteries were evaluated in terms of remaining patency and reported in percentages of normal.

A routine general post-mortem examination was performed on each of the sacrificed animals.

\section{RESULTS}

NEUROLOGICAL SYMPTOMS As a rule, the obliteration of the basilar artery alone did not produce detectable neurological symptoms in the majority of the animals; likewise, the occlusion of both common carotid arteries, with the basilar left patent, resulted in no deficit. The brains of such control animals were removed and were normal when studied in serial sections.

When, however, the basilar artery was occluded, together with one or both carotid arteries, symptoms and signs almost uniformly developed in the animals.

In the most severe form, these symptoms were expressed by either partial or complete decerebrate states which in a few animals did not lead to death. If one common carotid artery was left completely or partially patent, milder permanent neurological symptoms were detectable after recovery from the operation.

Such symptoms were exemplified by apathy, sluggishness, loss of self-care, and anorexia. The postural reflexes and placing, hopping, and righting reactions were often lost or diminished on one or other side. Asymmetries of the deep tendon jerks were often detectable. Some animals showed a tendency to drag one limb behind and to circle towards one or the other side. One animal was noted to neglect visual, acoustic, and tactile stimuli on his left side. No true convulsive phenomena or brain-stem signs were seen.

MACROSCOPIC CHANGES On post-mortem examination no changes were found in the internal organs as well as on the surface of the cerebrum. The most conspicuous cerebral lesions were observed in the deep regions of the centrum semiovale, and these consiste in well-defined and well-circumscribed patchy infarct 8 lying close to the ventricular walls. Some of these infarcts were present in the anterior limbs of the internal capsule at the level of the head of the caudate nucleus and in the corpus callosum and callosal radiations. In each one of the brains, all the infarcts present were of the same age and chronologically related to the duration of post-operative survival of the animal. The ligated segments of the carotid arteries as well as the basilar artery in situ were carefully studied in serial sections and most of them showed normal wall and endothelial lining. However, in two specimens there were single areas of subintimal proliferation covered by a layer of newly formed endothelium. No free mural thrombi were detected in any of the vessels.

Figure 1 exemplifies the typical findings in one of the brains; the basilar and the right common carotid arteries were completely occluded. Approximately $20 \%$ of the lumen of the left common carotid artery was patent. The post-operative course of the animal consisted of apathy, deviation of the head and eyes and circling to the right, and absence of tactile and visual placing reactions on the left side. There was $N$ striking indifference to visual, acoustic, and tactile stimuli on the left side.

MICROSCOPIC CHANGES Horizontal brain sections (Figs. 1a and 1b) show numerous periventricular 
areas of liquefaction necrosis, total loss of myelin, and corresponding patchy areas of gliosis (Fig. 1c). All of the lesions are of the same approximate age and show pronounced macrophage reaction and a tendency to follow the direction of periventricular vessels (Fig. 1d), all of which are either empty or contain fresh erythrocytes (Fig. 1d). There was only scanty and barely detectable peripheral astrocytosis. Lesions are present only in the right hemisphere (the side of the totally occluded common carotid artery) and their general distribution is reconstructed from the serial sections in Figure 1e.

Note the presence of a single cortical lesion in the depth of the sulcus ectosylvius as well as the infarcts in the internal capsule; the adjacent gray nuclei are completely spared. Another large lesion in the rostral portion of the callosal radiations encroaches upon the lateral aspect of the corpus callosum. Some of the multiple posteriorly located lesions interrupt the visual and auditory pathways, accounting for the specific sensory deficit described above.

Figure 2 demonstrates another example of periventricular leucomalacia in the brain of an animal with a post-operative course of 17 days and neurological symptoms of circling and deviation of the head and neck to the left plus loss of placing reactions and increase in tone on the right side.

The brain was cut in coronal sections. The multiple and patchy lesions can be compared with those seen in the previous example: tissue liquefaction, total disappearance of myelin and replacement by a pronounced lipid-laden macrophagic reaction, with a slight increase and swelling of the astrocytes at the periphery (Figs. 2a, 2b, 2c). Also, a well-defined necrotic area is present in the immediate vicinity of, but not involving, the caudate nucleus. In this brain, however, the infarcts are bilateral and more extensive and numerous in the left hemisphere, this as a result of bilateral pronounced stenosis of the common carotid arteries, more on the left $(30 \%$ patent $)$ than on the right (60\% patent) side.

The topographic distribution of the lesions is shown in Figure 2d. Again, the lesions are clearly related to the walls of the lateral cerebral ventricles. In addition, softenings are present in both internal capsules and in the splenium of the corpus callosum (torn, unfortunately, during removal of the brain). Most of the intraparenchymal vessels within the infarcts are congested and filled with fresh red blood cells (Fig. 2c) without erythrodiapedesis or occlusions of their lumina.

One more instance of patchy periventricular necrosis is shown in Fig. 3 where the softenings are present exclusively in the left hemisphere (Fig. 3a), i.e., on the side of the completely occluded common carotid artery. The vessel is shown at the point of occlusion (Fig. 3b).

As in the other cases, in the region occupied by cerebral softenings, there is pronounced disintegration of tissue with vasodilatation, microglial proliferation, and little or no astrogliosis. Again, no occluded vessels could be seen.

\section{SUMMARY OF THE PATHOLOGICAL FINDINGS}

The lesions were histologically represented by infarctions of the white matter with disintegration of myelin, pronounced softening and breakdown of tissue, and intense macrophage reaction accompanied by a slight or no increase of astrocytes at the periphery. The parenchyma between the lesions was remarkably well preserved and the grey matter was almost totally spared. In the two first cases, the infarcts were present in the immediate vicinity of the head of the caudate nucleus, completely sparing this structure. The same brains also showed partial involvement of the corpus callosum and of the internal capsule.

Almost all the lesions bore a close relationship to the lateral walls of the cerebral ventricles. In the first and the third brain, the lesions were seen only in the right and left hemisphere respectively (on the side of the occluded common carotid artery).

In the second brain, the softenings were bilateral, but more numerous and more intense in the left hemisphere, on the side of the severely stenosed carotid artery.

All the lesions in each brain were of comparable histological ages proportionate to the length of the post-operative course of the animal.

\section{DISCUSSION}

The presence of numerous foci of periventricular liquefaction necrosis in the brains of animals subjected to carotid and basilar artery ligations described above strongly suggests a haemodynamic element in the production of these lesions.

At first, however, the patchy characteristics and the apparently random distribution of the infarcts in the white matter suggested the effects of multiple emboli originating from the ligated arterial segments or internal organs. This possibility could safely be ruled out, since, in the first place, no source for emboli could be demonstrated (the results of the necropsy were normal and no disease was found in serial sections of the carotid artery). Secondly, all the necrotic foci were located in the periventricular white matter. Such a preferential localization of the 

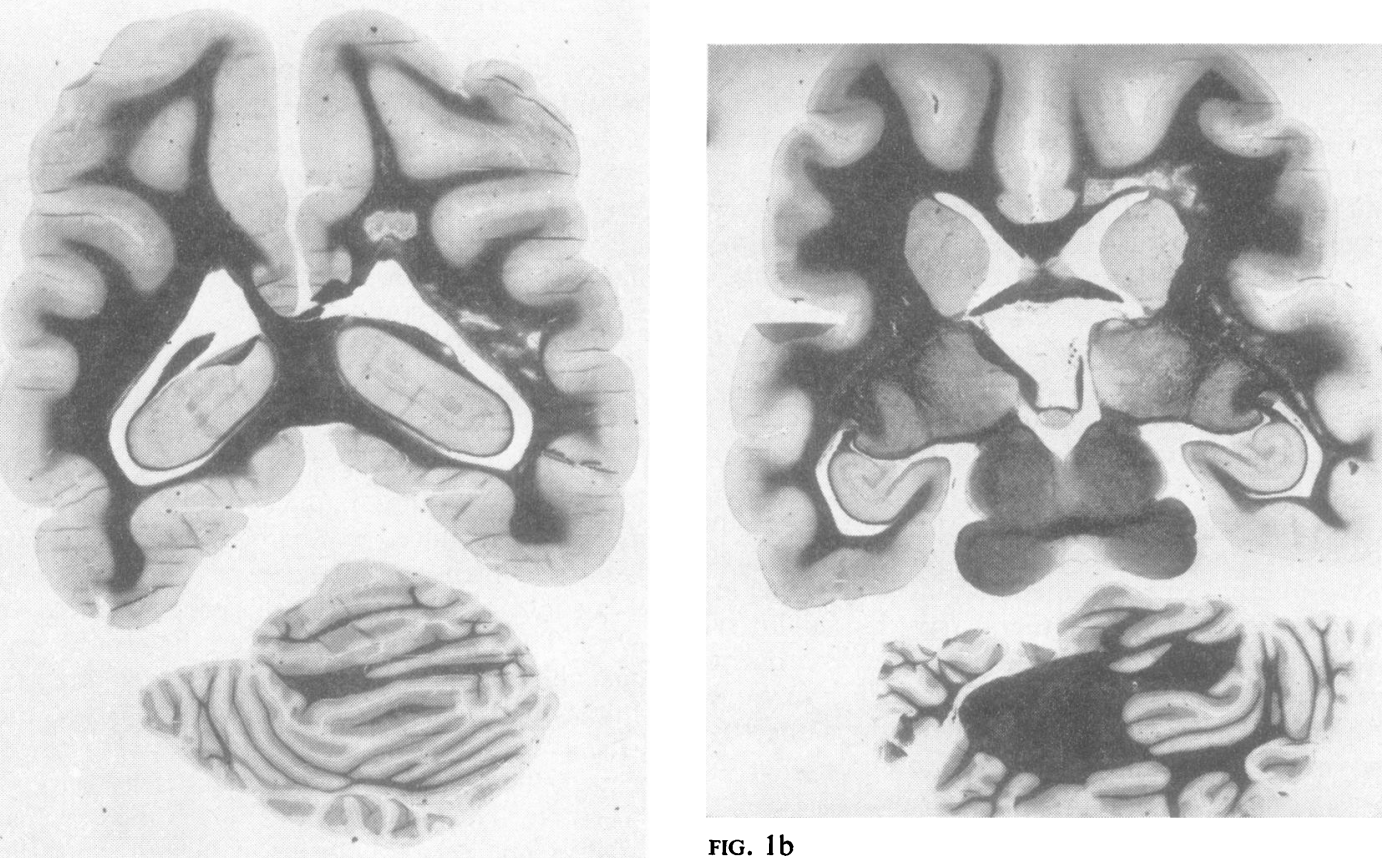

FIG. 1a

FIG. 1b
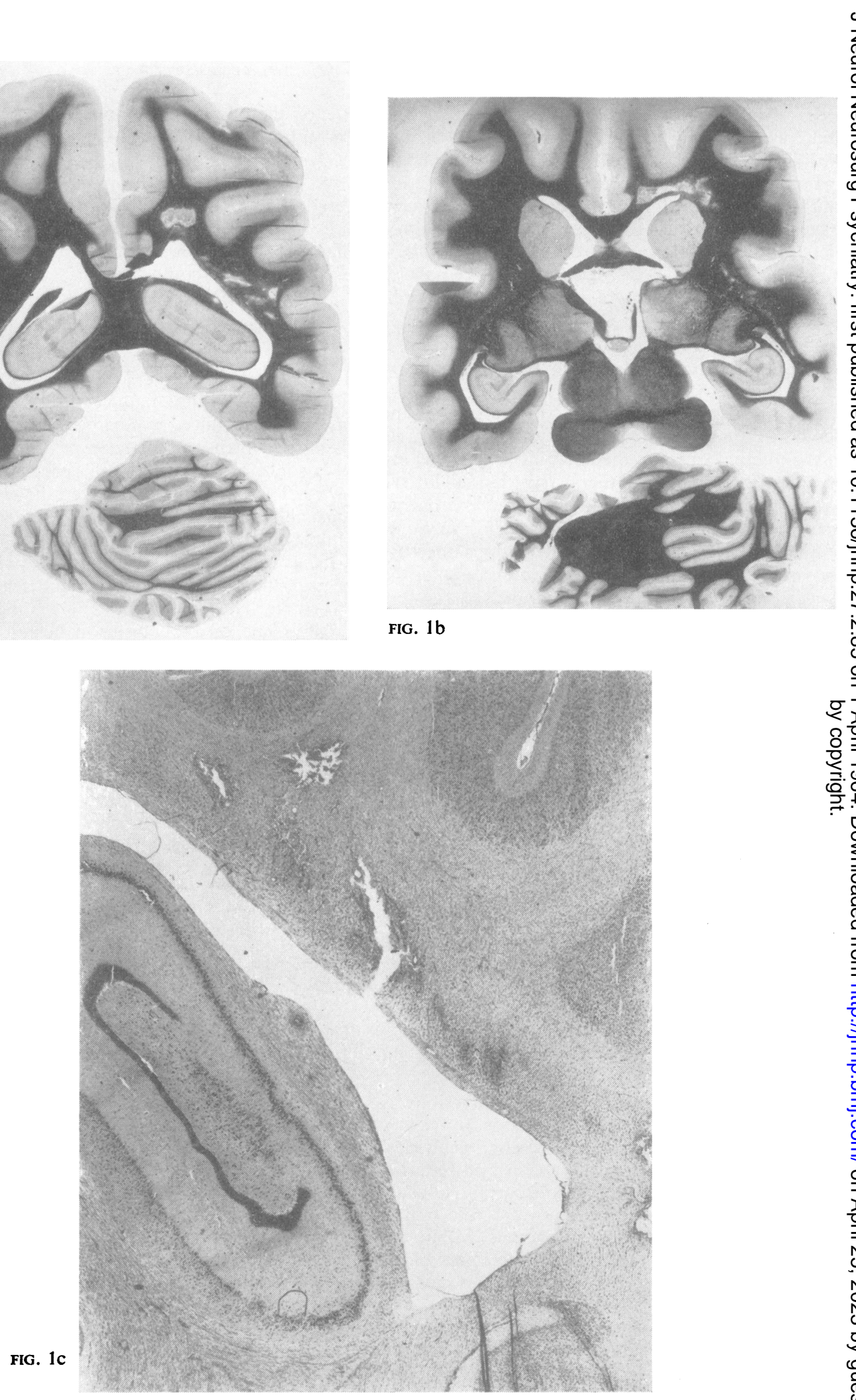


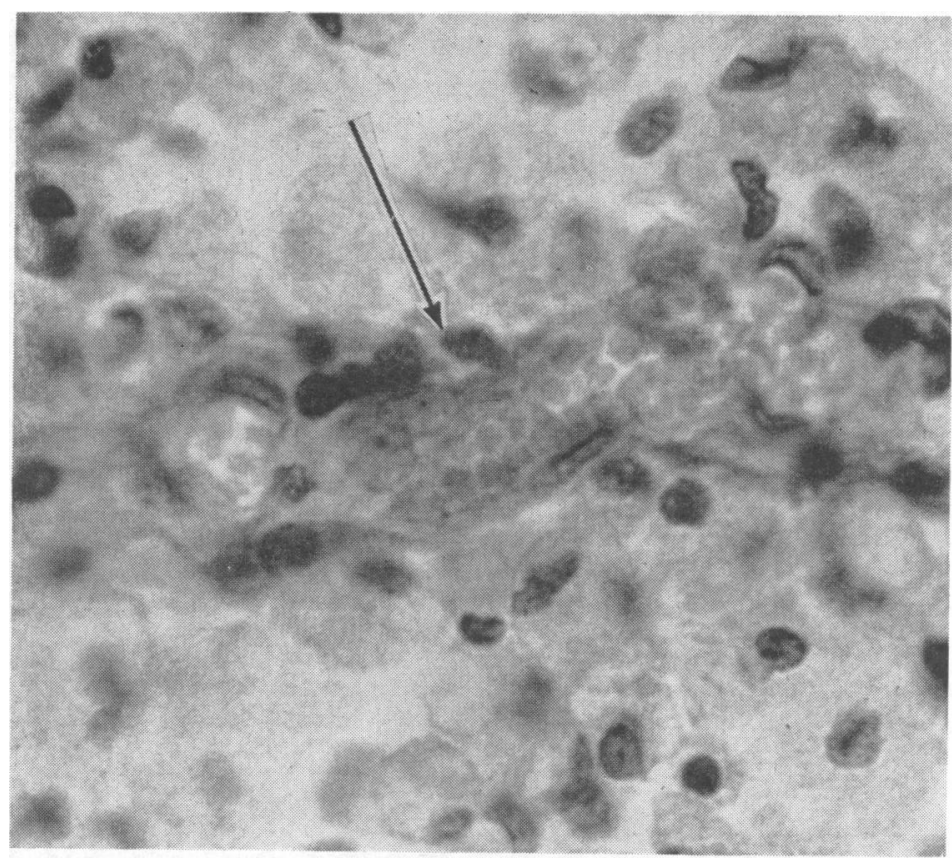

FIG. 1d

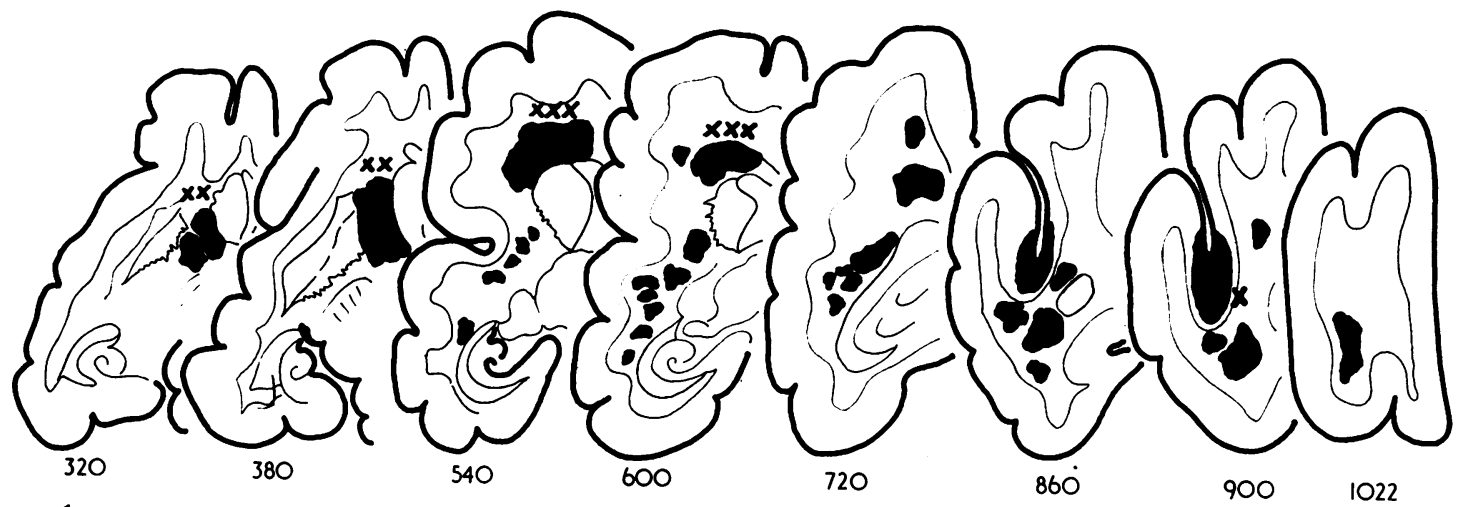

FIG. $1 \mathrm{e}$

FIG. 1. Brain of the animal C-6. Figs. $1 a$ and $1 b$. Two sections showing patchy periventricular softenings in the right hemisphere (Weigert stain on paraffin). Fig. lc. Low-power view of another section (Nissl stain): identical lesions showing intense gliosis. Fig. 1d. High-power view of a softening showing pronounced microgliosis and a vessel filled with erythrocytes (arrow). Fig. le. Diagrammatic template reconstruction of representative serial sections: $(X) A$ single cortical lesion; $(X X)$ pericaudate internal capsular lesions; $(X X X)$ softenings in the rostral portion of the corpus callosum. 


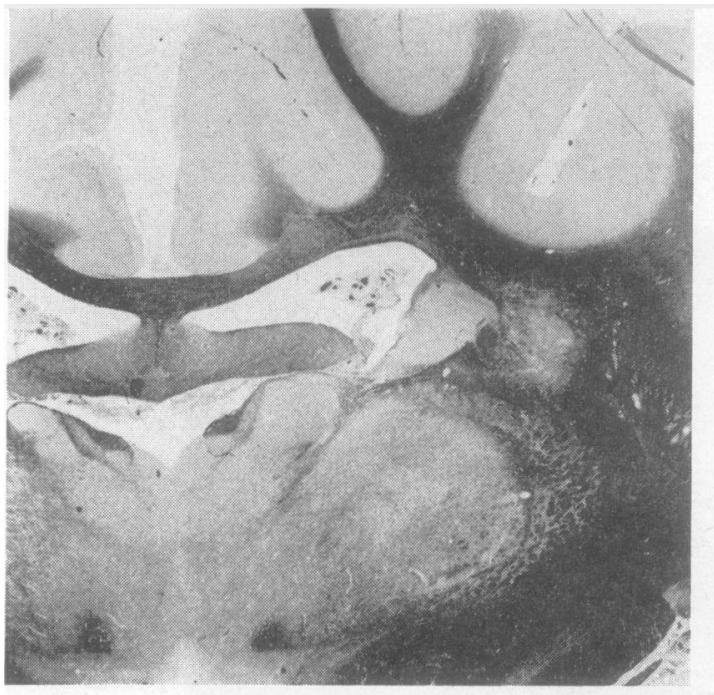

FIG. $2 a$

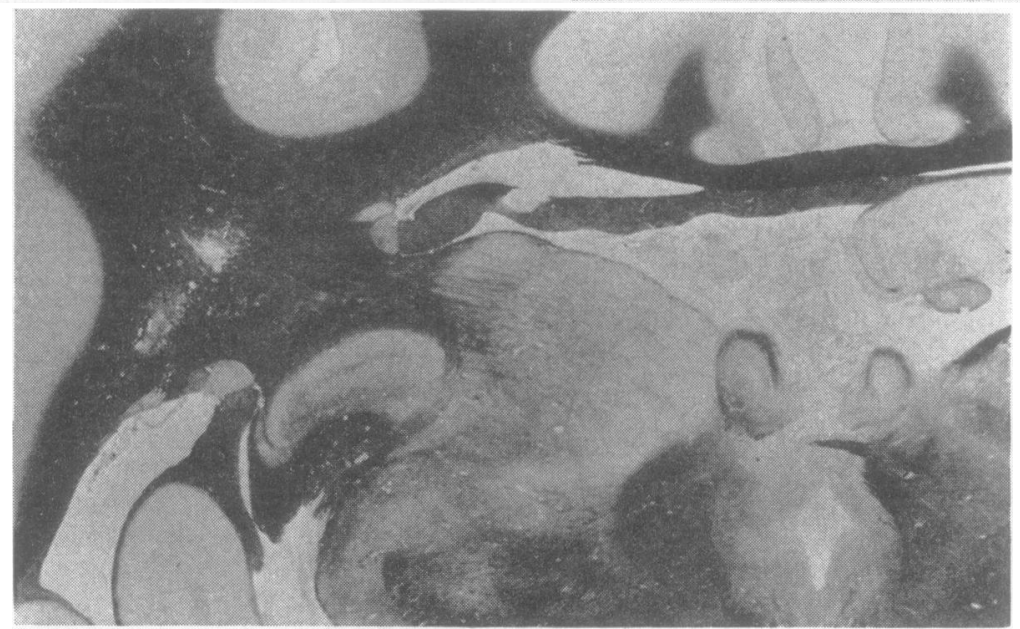

FIG. $2 b$

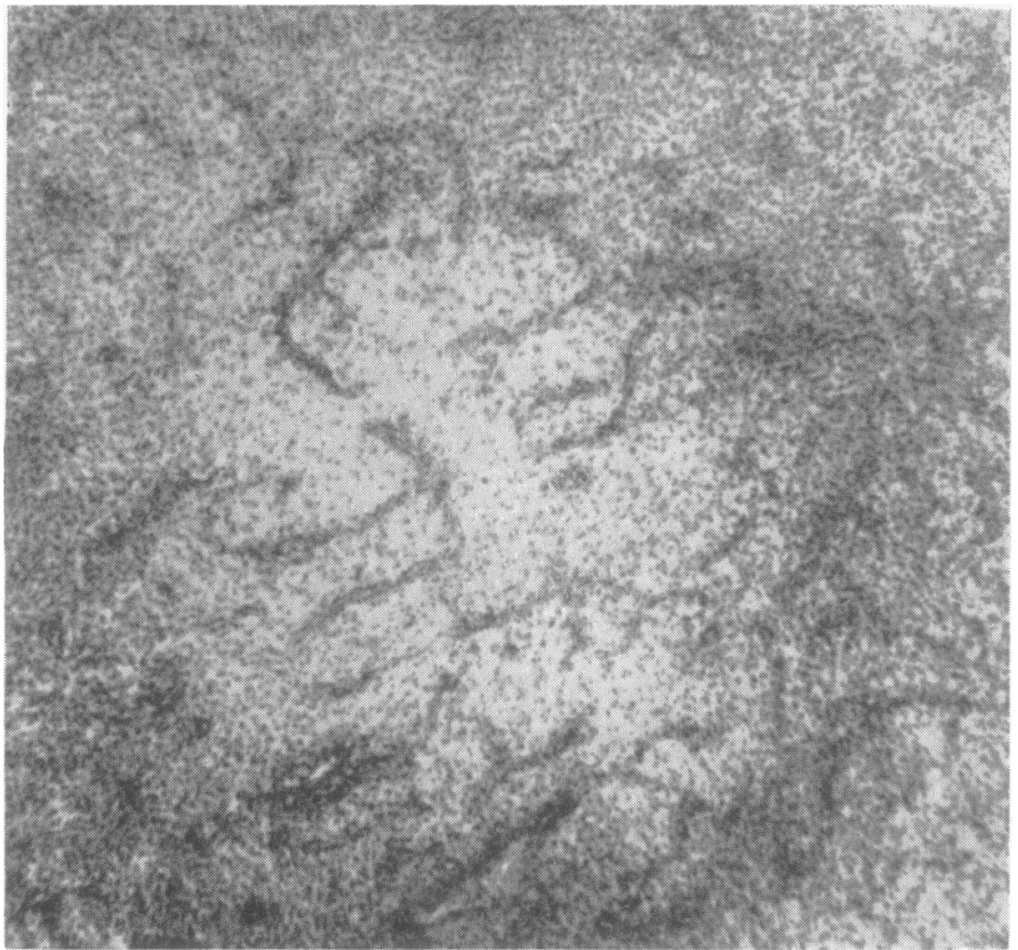

FIG. 2. Brain of the animal C-15. Figss. and $2 b$. Coronal sections showing pericaudate periventricular lesions (Loyez and Bielschon Plien stain on celloidin). Fig. 2c. Highers $p_{1}$ magnification. A softening (Nissl stain) shio central liquefaction of tissue, intense gliösis vascular congestion. Fig. $2 d$. Serial recetss. tion of the softenings. Note lesions in the inte capsules and in the splenium of the nxc callosum.

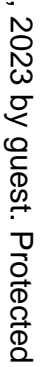




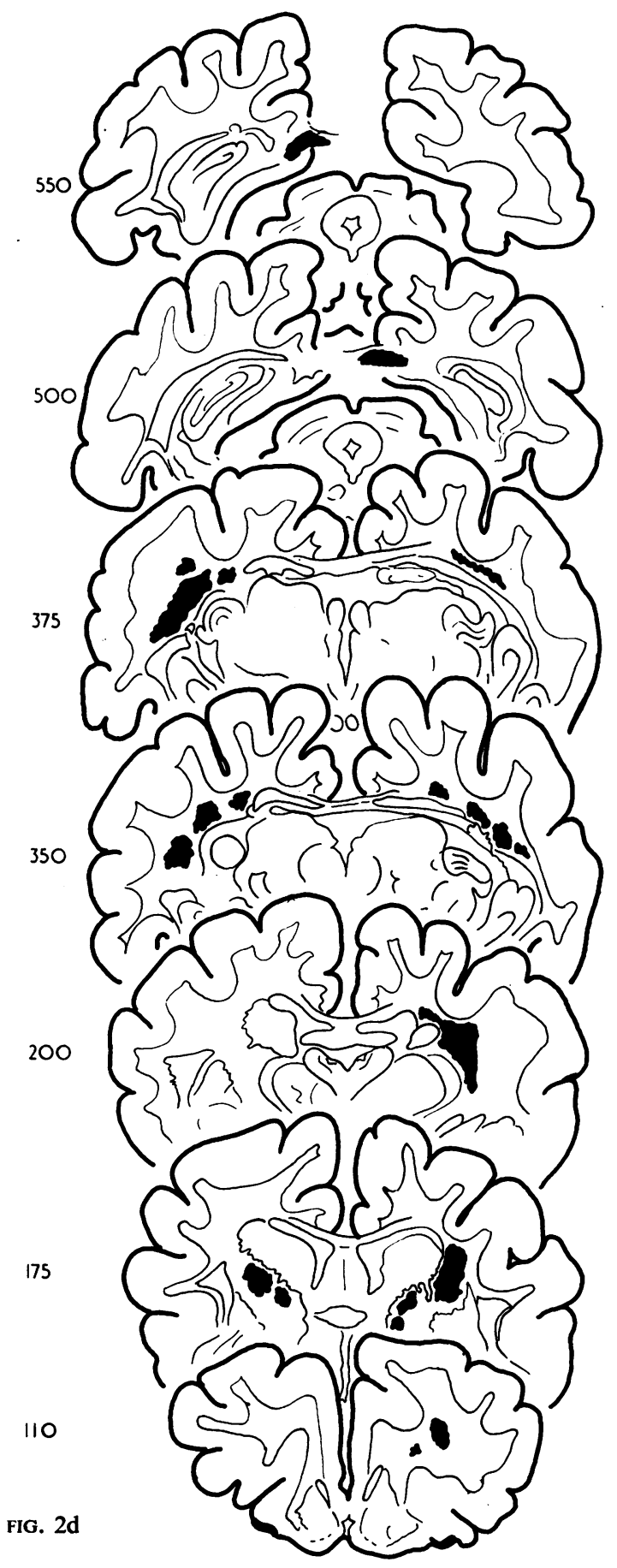

embolic fragments has never been demonstrated either in human or experimental pathology. Thirdly, in the first specimen all the infarcts were found exclusively in the hemisphere homolateral to the occluded carotid artery. In the second brain the infarcts were more intense and more numerous on the side of the vessel which was almost totally obliteraated. In the last reported case, necrotic foci were again found only in the hemisphere homolateral to the occluded common carotid artery. Finally, all the parenchymal vessels were carefully studied and were found to be patent or to contain fresh red blood cells (Fig. 1d).

The possibility of infection causing multiple brain abscesses could not be seriously entertained. Hence, for the reasons given above, the impairment of the blood flow resulting in stagnant tissue anoxia with subsequent coagulation and later liquefaction necrosis was thought to be the most likely explanation for the pathogenesis of the periventricular lesions in the animals. However, this preferential localization in the periventricular white matter presents a problem in cases in which a rational explanation of such regional vulnerability is desirable.

It is generally known that focal lesions of white matter occur in numerous conditions in which cerebral anoxia is primarily implicated or plays an important but not necessarily a direct role, for example, in carbon monoxide and potassium cyanide poisoning, nitrous oxide anaesthesia, a fall in systemic blood pressure in elderly individuals, or in experimental asphyxia (Hsü and Ch'eng, 1938; Hurst, 1940; Lewey and Drabkin, 1944; Morrison, 1946; Schwedenberg, 1959). In most of such reported cases, however, the lesions of the central white matter have a random distribution with no constant or definite relationship to the cerebral ventricles. Histologically, the leucomalacic foci are poorly circumscribed. It is possible that in the majority of the instances reported in the literature the anoxic state was not confined to the brain alone but was, in fact, generalized and affected many organs, of which the cardiopulmonary apparatus was most commonly involved, leading, in this way, to a self-perpetuating vicious cycle resulting eventually in cerebral lesions which were more extensive than those which would be expected if the anoxic state were confined to the brain alone.

Infarcts sometimes occurring in the periventricular white matter of adults with arteriosclerotic disease are almost always accompanied by lesions of the grey matter, particularly the cerebral cortex. Such foci of cortical necrosis are often located in the border zones of the arterial supply; an explanation for this preferential localization is offered elsewhere (Romanul and Abramowicz, 1964). 

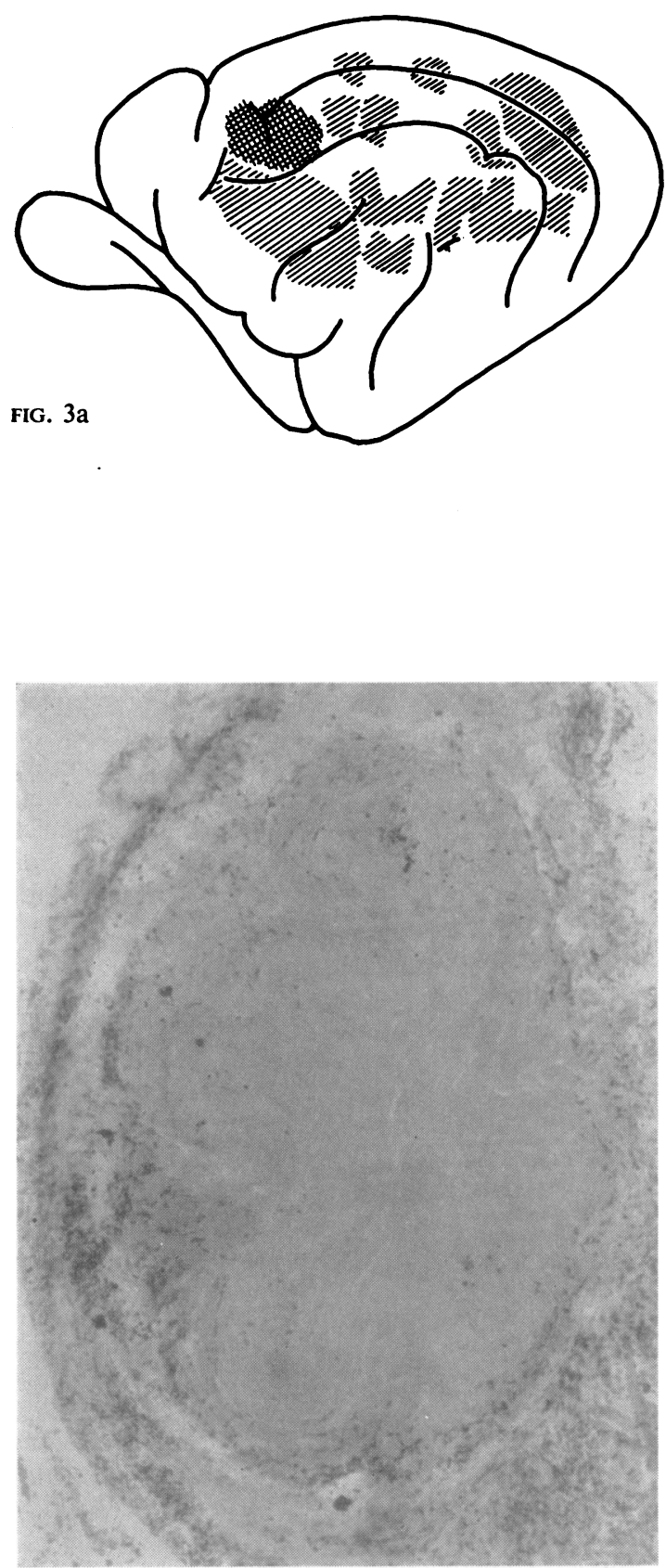

FIG. $3 b$

FIG. 3. Brain of the animal C-17. Fig. 3a. Distribution of the lesions in the white matter of the left hemisphere with a single cortical softening (cross-hatched) located on the border zone between the anterior and middle cerebral artery territories. Single hatched areas represent periventricular infarcts. Fig. 3b. Occluded left carotid artery.
Selective periventricular softenings, be it in tho form of fresh infarcts or in the form of old cystis lesions ('cystic degeneration'), are more commonlye found in the brains of infants and children who are subjected to birth injury. These lesions sometimese co-exist with cortical ulegyria, granular atrophys porencephalic cysts, or other scleroses and with foc를. of abnormal disposition of myelinated fibres: status marmoratus, and 'placques fibromyeliniques' of the basal ganglia and of the cerebral cortex.

Banker and Larroche (1962) made a pathologica study of a number of infant brains in which the most conspicuous and often the only lesions weres. represented by periventricular necroses of various ages (periventricular leucomalacia). In their casesీ there was, as a rule, a history of birth injury and ofterf of prematurity. Banker and Larroche offer anp interpretation of the formal genesis of such peri ventricular lesions which, in their cases, varied fromb coagulation and liquefaction necrosis to infarctions:with pronounced microglial and variable astroglia reactions and early cavitary changes. There was $\dot{a}$ correlation between the approximate duration of the peri- or post-natal distress of the patient and the histology of the lesions. Topographically, three major zones of involvement of the white matter were constantly noted: $1 \mathrm{~A}$ small area anterior $\overrightarrow{\mathrm{C}_{\vec{S}}}$ the anterior horn of the lateral ventricles; 2 the corona radiata; 3 the external and internal sagi⿺辶卬 layers of the temporal and occipital horn of E⿱⺈⿵⺆⿻二丨冂口⿱ lateral ventricles. After a review of the pertinente literature, Banker and Larroche favour the anoxi\& aetiology of periventricular leucomalacia, and ir support of their conclusion they quote the borde zone theory of Lindenberg and Spatz and state 'The periventricular lesions are located at the ver $\$$ termination of the major cerebral vessels in the border zones between anterior, middle, and posterio cerebral arteries'. They also mention that in the newborn the vessels subserving the periventriculat region are thin and poorly developed but give no experimental evidence for this assertion.

The histological appearance and the topographie distribution of the periventricular leucomalacia in. some of the cases reported by Banker and Larrochè resemble in most minute detail the pathologicas picture seen in the brains described in this paper.

The pathogenetic formulation offered by Banke? and Larroche is well founded and takes into con $\frac{7}{8}$ sideration the important contribution of Lindenberg. and Spatz regarding the border zones of arteria留 cerebral supply. It does not, however, adequately explain the problem presented by the preferentia localization of the lesions in the white matter of infants as contrasted with the predilection for the grey matter seen in the adult. The poor development 
of the arterial vessels subserving the periventricular region in the newborn is as yet to be proven experimentally.

These arteries, also called penetrating or 'radiating' arteries (Klosovskij, 1951), are characterized by their long course and the large diameter of their trunks. They are onto- and phylogenetically probably the oldest arteries of the mammalian brain and are part of the primitive 'arteriovenous units' which in some lower organisms, e.g., the green lizard, the opossum, the kangaroo (Wislocki and Campbell, 1937; Klosovskij, 1956), represent the main and sometimes the only sources of intraparenchymal cerebral blood supply.

This system of arteriovenous units persists in a more or less conspicuous form in all the vertebrates and is recognizable even in adult man. The long straight penetrating arteries can be plainly seen even on gross transversal brain sections and in injected specimens. These arteries stream towards the external walls of the lateral cerebral ventricles where they converge; their convergence is particularly circumscribed at the level of the anterior horn of the lateral ventricles (Lazorthes, 1961).

With the progressive development of the brain, and particularly with its passage from a relatively smooth (lissencephalic) to the more intricate fissurated and sulcated form seen most conspicuously in adult man, a second fundamental system of vascular patterns becomes more evident and superimposed, as it were, upon the pre-existing system of arteriovenous units. This newer system consists of a network-like arrangement of innumerable collateral channels stemming from the more recently developed intracortical and subcortical vessels, all of them shorter and thinner than the primitive long penetrating arteries. The latter vessels, as stated above, persist, however, in an almost unchanged form throughout the phylo- and ontogenetic development of the brain. Embryologically, these arteries, together with the capillary arteriovenous plexuses and the emerging straight veins, are thus the remnants of the primitive arterial venous units which originally supplied the cerebral matrix zone and remain in their unchanged position throughout the entire embryological sequence (Klosovskij, 1951 and 1956). They are, in addition, characterized by the paucity and feebleness of their collateral branches (Campbell, 1938; Klosovskij, 1956), which, according to Lazorthes (1961), are never authentic anastomoses.

The cerebral vascular system of a mature cat resembles in many details the arrangement seen in the human foetus near the term of gestation. In particular, the periventricular region is subserved by straight long penetrating arteries, while the network- like newer system of collaterals is still relatively poorly developed. Furthermore, in analogy to the human foetus, the leptomeningeal arterial system of anastomoses is represented by a series of wide arterial channels comparable in the sizes of their diameters to the terminal branches of the three major cerebral arteries which these anastomoses unite. There is a relative paucity or absence of the highly differentiated, intricate and incomplete arterial anastomotic loops characteristically found in the depth of the sulci in the mature human cerebrum (Klosovskij, 1951; Vander Eecken, 1959). The maturation with the increase in the number of gyri and sulci in the adult human cerebrum, as contrasted with the lower animals and human foetuses, proceeds pari passu with the major differentiation and breakdown in the completeness of the anastomotic connexions of the cerebral convexity. These processes have the greatest expression in those areas of the neocortex which are peculiar to the mature human brain.

It is, therefore, surmised that in contradistinction to the lower animals and human foetuses, the zones of major haemodynamic cerebral vulnerability in the mature human brain are principally represented by these highly differentiated cortical areas supplied by the incomplete and, hence, highly differentiated anastomotic loops which are prone to favour the occurrence of regional stasis due to various regional or systemic causes. This situation is reversed in the lower animals, for example, the hare or the cat, and in human foetuses where the ampleness and the completeness of the leptomeningeal anastomoses safeguards the relative integrity of the superficial cerebral regions.

It would, therefore, appear that because of the relative lack of collateral branchings and the absence of true anastomoses in the vessels subserving the periventricular white matter in animals and in man the possibility of collateral blood supply in those regions is extremely small, if ever possible. Moreover, in the territory where one deep area of supply of a major cerebral artery lies adjacent to another, i.e., roughly in the lateral anterior superior portion of the lateral ventricles for the anterior and the middle cerebral arteries, there exists a functional and anatomical separation of the blood flow. If one of such territories falls short of the expected blood supply, one effective means of collateral blood supply would be provided by a reversal of blood flow via the periventricular capillary plexus. Such a reversal of flow is obviously a theoretical possibility but never clearly demonstrated experimentally and would be, at its best, slow and relatively inefficient. For practical purposes, therefore, the straight penetrating arteries reaching the periventricular 
region can be considered to be functionally and anatomically terminal, particularly when the intravascular cerebral blood pressure a tergo decreases due to a variety of reasons. For example, the exclusion of a single arteriovenous unit by obliteration of a penetrating artery in the opossum leads to a circumscribed area of cerebral infarction in the region of the distal capillary network in a radius of approximately $25 \mathrm{~m}$. in diameter (Klosovskij, 1956). In this animal, the arteriovenous unit is the principal constituent of the cerebral vascular system (Wislocki and Campbell, 1937).

In summary, then, the extraordinary length and the relative absence of anastomoses in the penetrating periventricular arteries renders them anatomically terminal and predisposes the territories of their supply to major vulnerability in conditions of impaired blood flow, with resulting foci of softening in the distalmost portions of the arterial supply analogous to the situation described in the opossum. In the relatively lissencephalic (smooth) brains of lower mammals and of the human foetus the leptomeningeal anastomoses are represented essentially by end-to-end straight connexions between the still sparse branches of the three major cerebral arteries, permitting a good collateral blood supply between one and another pial artery. The major stress in such circumstances will fall upon the penetrating and functionally terminal straight arteries deprived of significant collateral connexions.

Some of these long penetrating arteries, in addition, originate from the leptomeningeal branches of the middle cerebral artery while others stem from similar branches of the anterior and posterior cerebral arteries; still others initiate their course towards the ventricles from the leptomeningeal border zones of these three vessels. An even greater haemodynamic stress will, therefore, occur where such territories separate (the deep cerebral border zone), that is, in the white matter of the periventricular region. This region is doubly vulnerable, as it were, in virtue of the functional and anatomical terminal character of its vessels and of the origin of these vessels from the leptomeningeal border zone. In the specific instance of experimentally curtailed cerebral blood supply secondary to the narrowing or occlusion of the carotid and basilar artery, the periventricular regions suffered an elective damage because, in addition to their location in the terminal zones of the vascular supply, they were also situated at the border zones of supply of the three major cerebral arteries.

In the brains described, however, in addition to the purely periventricular lesions, softenings were found in the anterior portions of the internal capsule at the level of the head of the caudate nucleus (Fig. 2a). This particular location did not a first seem amenable to my pathogenetic interpreta tion. The lenticulo-striate branches of the middle cerebral artery supply this area of the internal capsule together with the adjacent portions of the putamen? and the caudate nucleus. The total sparing of thes gray matter of those nuclear aggregates was no readily explicable, therefore, by the known an atomical data. On the other hand, both caudate and. putamen are often infarcted when the occlusion of the main trunk of the middle cerebral artery occurso proximal to the origin of the lenticulo-striates

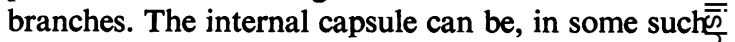
instances, selectively spared and this fact migh $\frac{\mathbb{Q}}{2}$ suggest a special anatomical arrangement of the vessels supplying this region.

Until recently, the lenticulo-striate arteries were $\overrightarrow{0}$ all thought to be truly terminal vessels. Ismailova in $\overrightarrow{-}$ 1948 and Ogniev in 1950 (quoted by Kukushkina, $\omega$ 1956) have demonstrated arterial anastomoses to exist between these vessels and the arteries penetra 0 ing from the cerebral convexity. These authors make no mention of the relative number or the sizes of such anastomotic channels. More recently, Dinsdale of (1963), has studied a number of human brainso injected with barium and gelatin and has demonstrat- ed anastomotic vessels interconnecting the penetrat $\rightarrow$ ing arteries from the upper brain convexity with the lenticulo-striate branches supplying the head of caudate nucleus. He felt that these anastomoses wees comparable in diameter to the large penetratifig. arteries supplying the periventricular regions. Such vessels seem to be fairly numerous but their exact number has not been as yet estimated. Probablyo some individual variations from brain to braino account for the inability of some authors to demon- $\bar{Q}$ strate these anastomoses (Alexander, quoted by $\overrightarrow{\vec{\rho}}$ Kukushkina, 1956). When they are present, however, $\Xi$ they can provide an important collateral pathway? safeguarding the structural integrity of the basalo ganglia in circumstances of reduced cerebral blood supply. These anastomoses provide, therefore, aร link between the vessels of the outer cerebralconvexity supplied by the middle cerebral artery and 3 . the vessels of the lenticulo-striate system originating more proximally from the trunk of the same artery, a border zone between two parts of the distribution of the middle cerebral artery. It is not possible to state that the internal capsule does not possess theo similar anastomotic channels, although they have not been demonstrated in the study by Dinsdale. $N$ Theoretically, at least, the anterior segment of then internal capsule between the head of the caudateN nucleus and the mid portion of the putamen can $\omega$ suffer a selective involvement similar to that seen in the periventricular region. An anatomical investiga- 
tion of this problem is now in progress and will be reported at a later date.

\section{SUMMARY}

Following experimental obliteration of the basilar artery and subsequent narrowing or closure of one or both common carotid arteries in mature cats, the animals exhibited chronic neurological symptoms indicative of cerebral deficit. The brains of some of the animals cut in serial sections showed selective involvement of periventricular white matter by multiple, patchy and well-defined foci of liquefaction necrosis although the cerebral grey matter was almost completely preserved. The resemblance of these infarcts to the lesions seen in brains of infants and children following anoxia and cerebral palsy suggested that a haemodynamic factor is implicated in the mechanism producing such necrotic foci. Selective involvement of the white matter lying close to the cerebral ventricles can be explained on the basis of recent studies in the field of comparative and developmental anatomy of the cerebral vascular system. In lower animals and in immature human brains the main blood supply of the deep cerebral white matter derives from a system of primitive 'arteriovenous units' whose straight and long penetrating arteries have few collaterals and no authentic anastomoses; they are essentially terminal vessels rendering the territory of their supply vulnerable to ischaemia produced by a variety of regional or systemic haemodynamic derangements. In addition, functional and anatomical separation of such arteries derived from the three major cerebral arteries in certain regions of the periventricular white matter (deep cerebral border zone) accounts for an additional element favouring the selective vulnerability of periventricular space; a concept of 'double vulnerability' is, therefore, proposed. The preservation of the gray matter can be accounted for by the presence in the immature and relatively smooth brains of ample and still poorly differentiated leptomeningeal anastomotic channels capable of transporting and shunting large quantities of blood. This is in contrast to the mature, richly sulcated human cerebrum where the leptomeningeal anastomotic network is highly differentiated into a system of feeble and incomplete arterial loops favouring local stasis and consequent preferential involvement of the cortical and/or subcortical border zone territories of the three major cerebral arteries.

The author is indebted to Dr. T. E. Twitchell for the use of his laboratory facilities, and to Drs. P. I Yakovlev, Norman Geschwind, and Joseph Segarra for helpful criticisms in reviewing the draft.

\section{REFERENCES}

Banker, B. Q., and Larroche, J. (1962). Arch. Neurol. (Chic.), 7, 386.

Benda, C. E. (1952). In Proceedings of the 1st international Congress of Neuropathology, Rome, edited by Rosenberg and Sellier, Turin.

Campbell, A. C. P. (1938). Res. Publ. Ass. nerv. ment. Dis., 18, 69.

Courville, C. B. (1953). Contributions to the Study of Cerebral Anoxia. San Lucas Press, Los Angeles.

Dinsdale, H. (1963). Personal communication.

Greenfield, J. G. (1958). In Neuropathology. Arnold, London.

Hsü, Y. K., and Ch 'eng, Y. L. (1938). Brain, 61, 384.

Hurst, E. W. (1940). Aust. J. exp. Biol. med. Sci., 18, 201.

Klosovskij, B. N. (1951). Circulation of Blood in the Brain (in Russian). Medgiz, Moscow.

(1956). Circulatory Impairments in Cerebral Diseases (in Russian). Medgiz, Moscow.

Kukushkina, V. P. (1956). Ibid.

Lazorthes, G. (1961). Vascularisation et Circulation Cérébrales. Masson, Paris.

Lewey, F. H., and Drabkin, D. L. (1944). Amer. J. med. Sci., 208, 502

Morrison, L. R. (1946). Arch. Neurol. Psychiat. (Chic.), 55, 1.

Norman, R. M. (1949). Brain, 72, 83.

- (1958). In Neuropathology. Arnold, London.

- , and Urich, H. (1959). Recent Progr. Psychiat., 3, 185.

Romanul, F., and Abramowicz, A. (Arch. Neurol., in press).

Schwartz, P. L. (1961). Birth Injuries in the Newborn. Karger, Basel, New York.

Schwedenberg, T. H. (1959). J. Neuropath. exp. Neurol., 18, 597.

Vander Eecken, H. M. (1959). The Anastomoses Between the Leptomeningeal Arteries of the Brain. Thomas, Springfield, Illinois.

Virchow, R. (1867). Quoted by Banker and Larroche.

Wislocki, G. B., and Campbell, A. C. P. (1937). Anat. Rec., 67, 177.

Zülch, K. J. (1961). Acta neurochir. (Wien), suppl., 7, p. 51.

Virchow, R. (1867). Virchows Arch. path. Anat., 38, 129. 